\title{
Philosophiques
}

\section{Probabilités subjectives et métaphysique}

\section{Anna Longo}

Volume 47, numéro 2, automne 2020

URI : https://id.erudit.org/iderudit/1075132ar

DOI : https://doi.org/10.7202/1075132ar

Aller au sommaire du numéro

Éditeur(s)

Société de philosophie du Québec

ISSN

0316-2923 (imprimé)

1492-1391 (numérique)

Découvrir la revue

Citer ce document

Longo, A. (2020). Probabilités subjectives et métaphysique. Philosophiques, 47(2), 427-436. https://doi.org/10.7202/1075132ar d'utilisation que vous pouvez consulter en ligne.

https://apropos.erudit.org/fr/usagers/politique-dutilisation/ 


\title{
Probabilités subjectives et métaphysique
}

\author{
ANNA LONGO \\ Université de Paris 1, Panthéon-Sorbonne
}

\begin{abstract}
Certes, on ne pourra pas admettre le déterminisme, on ne pourra pas admettre l' «existence» dans le prétendu royaume de ténèbres et mystère, des lois immuables et nécessaires régulant l'univers, et on ne pourra pas admettre que cela soit vrai pour la raison simple que, à la lumière de notre logique, cela est dépourvu d'une signification quelconque. La science, entendue comme une activité de découvertes de vérités absolues, reste ainsi, naturellement, au chômage par manque de vérités absolues. Cependant, ceci ne conduit pas à une destruction de la science, mais mène seulement à une conception différente de la science $^{1}$.
\end{abstract}

Ainsi s'exprimait Bruno de Finetti dans Probabilismo, l'un des textes fondateurs de la théorie subjective des probabilités qui a inspiré le QBisme ${ }^{2}$ (bayésianisme quantique), voire l'interprétation de la mécanique quantique que Michel Bitbol partage et considère comme s'accordant avec l'épistémologie qu'il propose dans Maintenant la finitude. Or ma question est la suivante: le "corrélationisme radical» de Bitbol est-il assez radical ou devrait-on plutôt considérer le "probabilisme radical ${ }^{3}$ " proposé par Jeffrey en suivant de Finetti et Ramsey, comme à la fois plus cohérent et plus efficace du point de vue d'une explication antimétaphysique du succès de la science actuelle? Le probabilisme radical ne nous permet-il pas de dévoiler l'insuffisance du critère de cohérence proposé par Bitbol, ce qui rendrait incertaine sa victoire contre le matérialiste spéculatif? Comme je vais le montrer, tout en partant d'une position assez proche, les conclusions de Bitbol et des bayésiens subjectivistes diffèrent quant à la radicalité des propos antimétaphysiques. Comme de Finetti le soutient:

Je ne cherche pas à savoir pourquoi le FAIT que je prévois se réalisera, mais pourquoi JE prévois que le fait va se réaliser. Ce ne sont plus les faits qui ont besoin d'une cause pour se produire: c'est notre esprit qui trouve commode

1. Bruno de Finetti, "Probabilismo. Saggio critico sulla teoria delle probabilità e sul valore della scienza » (1931), in La logica dell'incerto, Milano, Il Saggiatore, 1989, p. 3-4. Ma traduction.

2. Carlton M. Caves, Christopher A. Fuchs, and Rüdiger Schack, «Unknown Quantum States: the Quantum de Finetti Representation ", in Journal of Mathematical Physics, 43, 4537 (2002).

3. Richard Jeffrey, "De Finetti's Radical Probabilism», in de Finetti, Probabilità e induzione, Bologna, CLUEB, 1993, p. 263-275. Richard Jeffrey, «Radical Probabilism (Prospectus for a User's Manual) ", in E. Villanueva (dir.), Rationality in Epistemology, Ridgeview Publ. Co., 1992, p. 193-204.

PHILOSOPHIQUES 47/2 - Automne 2020, p. 427-436 
d'imaginer des rapports causaux afin de les expliquer, de les coordonner et d'en rendre la prédiction possible ${ }^{4}$.

Cette position semble s'accorder avec celle de Bitbol, lorsqu'il explique: "Ce n'est pas qu'un “monde-de-faits” soit intrinsèquement conforme à une loi de l'univers; c'est seulement que, dans le cours de notre recherche, nous appliquions constamment, nécessairement, le principe régulateur de l'entendement qu'est la "loi de liaison de la cause et de l'effet", et que nous anticipions l'unité des phénomènes sous le présupposé de cette loi comme si elle était universelle ${ }^{5}$.» À partir du constat que, sauf en admettant quelque raison métaphysique, la méthode inductive ne peut garantir la nécessité des prévisions des événements futurs, la meilleure solution au problème de Hume semble l'attitude pragmatiste: les lois de la physique sont des hypothèses d'action qui permettent de s'attendre à des résultats. Il s'agit d'habitudes sélectionnées qui permettent de s’orienter et de prendre des décisions efficaces dans une réalité incertaine, il s'agit de stratégies de pari qui permettent de calculer la probabilité du succès des opérations qu'on met en place afin de rendre des expériences possibles. Le QBiste, comme Bitbol l'explique, reconnaît la validité de cette approche, et «il considère cet ordre apparent comme le travestissement superficiel dont un univers, peut-être désordonné, est recouvert par un système ordonné de comportements présents, et de paris sur l'avenir: le système des paris grâce auxquels des agents d'échelle macroscopique organisent par avance les conditions dynamiques de leur survie au cœur d'un environnement pouvant être chaotique ${ }^{\star}$. De cette manière, on ne doit pas se soucier de l'ordre métaphysique du monde, mais seulement de la probabilité de succès de nos paris (décisions d'action) et des raisons qui peuvent nous donner plus ou moins confiance dans des opérations techniques, conduites dans un cadre expérimental nous permettant d'espérer voir un résultat se réaliser. Chez les subjectivistes, en théorie des probabilités, comme de Finetti et les QBistes, la méthode des paris est employée pour mesurer le degré de croyance personnelle en une hypothèse comportant une décision d'action lorsqu'aucune probabilité logique, a priori, n’est admise pour évaluer la vérité universelle de l'implication entre état des faits observé et inférence prédictive ${ }^{7}$. Cette méthode est l'une des manières ${ }^{8}$ de quantifier la confiance personnelle dans une prédiction relative

4. De Finetti, op. cit. p. 4. Ma traduction

5. Michel Bitbol, Maintenant la finitude. Peut-on penser l'absolu?, Paris, Flammarion, 2019, p. 221.

6. Michel Bitbol, op. cit., p. 241.

7. Cette dernière est la position «objective" de Carnap et de Keynes qui considèrent la probabilité comme exprimant la relation logique entre une prémisse et une conséquence. Cette interprétation des probabilités est à l'origine de la théorie classique de la décision rationnelle critiquée par De Finetti et aussi par Bitbol pour des raisons similaires.

8. D'autres manières de mesure du degré de croyance ont été proposées, par exemple par Ramsey qui considère les décisions qui permettent de réaliser une utilité dans un système cohérent de préférences. 
à un contexte précis de décision (comme parier sur la couleur de la prochaine boule tirée d'une urne dont on ne connaît pas le contenu, mais dont on a pu observer un certain nombre de tirages) afin d'en vérifier la cohérence $^{9}$. Ce qui compte est que cette méthode des paris, dont Bitbol utilise à plusieurs reprises le vocabulaire, présuppose que la probabilité ne se réfère pas à l'ignorance de faits qui seraient déjà en quelque sorte déterminés, mais

la probabilité a un caractère essentiellement relatif; ceci suffit pour détruire le mythe d'une probabilité vraie, qui existe dans le "royaume de ténèbres et mystère » de la réalité suprasensible, ceci suffit pour faire collapser cette espèce de semi-déterminisme considérant deux cas également probables comme deux cas entre lesquels la nature serait encore libre de choisir et qui, puisqu'ils ne présentent aucune caractéristique pour se rendre préférables, forcent la nature dans l'indécision de l'âne de Buridan ${ }^{10}$.

Nos paris dépendent du contexte, et la probabilité n'exprime que le degré de croyance dans une hypothèse d'action à partir de l'information qu'on a pu collecter relativement à notre position située. Sur cette interprétation de la probabilité, comme portant sur la manière dont on va à l'encontre du réel en s'attendant à certaines réponses, s'accordent à la fois le QBiste et le corrélationiste radical:

Les probabilités de la mécanique quantique, comme toute probabilité, expriment la disposition d'un agent à s'en servir pour placer des paris. Cette disposition est fondée sur un jugement personnel qui est informé par les croyances de l'agent. Nous avions remarqué que la position Qbiste, selon laquelle les états quantiques sont des jugements personnels d'un agent, est une conséquence de la perspective subjective des probabilités qui a été exprimée d'une manière éloquente par Bruno de Finetti ${ }^{11}$.

Autrement dit, ici comme ailleurs, la limite alléguée de la connaissance ne se convertit pas en description du connu-tel-qu'il-est, mais en prescription sur la manière optimale de s'attendre à ce qui va se manifester. [...] Ainsi, loin de consister en un dévoilement progressif du réel à travers l'autorévélation des bornes de sa connaissance, l'histoire de la physique de Newton à Schrödinger se lit comme une succession de directives (ou de paris) pour devancer l'apparaitre de façon de plus en plus générale, à travers un jeu dialectique où chaque proposition subséquente est informée par l'acte de nier et d'élargir la proposition précédente ${ }^{12}$.

9. L'argument du pari hollandais (Dutch book) montre que si les croyances subjectives ne respectent pas les lois de la probabilité, un joueur peut être victime d'une martingale. La méthode des paris n'est pas la seule employée pour établir le même critère de cohérence; voir, par exemple Frank P. Ramsey, Truth and Probability, 1926.

10. Bruno de Finetti, op. cit., p. 16. Ma traduction.

11. Christopher A. Fuchs, N. David Mermin, and Rüdiger Schack, «An Introduction to QBism with an Application to the Locality of Quantum Mechanics ", in American Journal of Physics 82, 749 (2014), p. 753. Ma traduction

12. Michel Bitbol, op. cit., p. 246. 
Il y a cependant un point fondamental où le probabiliste radical et le corrélationiste radical divergent. Ce point concerne le critère de cohérence. Pour Bitbol, la cohérence reflète l'exigence de non-contradiction entre le dire et le faire du sujet connaissant, c'est-à-dire l'accord entre «l'acte présent de les penser, le fait présent de les éprouver comme cohérents ${ }^{13}$ ». La cohérence requise est celle du cercle où les conditions transcendantales de la connaissance présente rendent possible un contenu qui ne contredit pas l'explication de la genèse naturelle de ces conditions mêmes, cette cohérence serait ce qui permet de prendre des décisions qui s'alignent avec le processus de (co)création en quoi consisterait le réel, ce qu'on éprouve comme «vie» ou acte originaire répété dans toute évolution ou production de connaissance. Ainsi, l'épistémologie naturalisante et l'épistémologie transcendantale «opèrent comme deux temps d'un même processus consistant, tantôt à faire ressortir les conditions de possibilité d'un domaine de la connaissance établi depuis longtemps, tantôt à remodeler la relation constitutive de ce domaine afin d'engendrer un domaine de connaissance nouveau, voire révolutionnaire ${ }^{14} »$. Les deux épistémologies sont, l'une normative et a priori - elle prescrit les opérations à effectuer -, et l'autre, descriptive et a posteriori - elle décrit l'étant par rapport aux pratiques acquises et retrace l'histoire à travers laquelle ces habitudes inductives évoluent. En outre, il faut « une stratégie prédictive, prolongée par une procédure de décision, conforme à la conception de la connaissance vers laquelle convergent ces deux types d'épistémologies ${ }^{15}$ »; ces stratégies «indiquent quoi faire, comment se comporter, sous des hypothèses conformes aux normes et aux procédures respectivement indiquées par les deux genres d'épistémologies ${ }^{16}{ }$. Or le point de vue corrélationiste est interne au cercle, puisque les actions et les décisions sont vécues dans la pratique effective plutôt qu'elles ne constituent un modèle idéal qui viendrait à s'imposer de l'extérieur, comme c'est le cas dans les théories de la décision rationnelle. Dans ces dernières, élaborées à partir d'une conception objective de la probabilité, la décision dépend d'une définition a priori de la rationalité qui vise à la satisfaction d'utilités préétablies et qui, de fait, force à des schémas d'action qu'on pourrait dire artificiels et incapables d'évolution «vivante».

Notons que, dans ce schéma décisionnel conforme au paradigme réaliste, tous les paramètres sont tenus pour intrinsèquement déterminés, même s'ils sont incomplètement connus. Les propriétés de l'environnement sont considérées comme des déterminations intrinsèques à propos desquelles les agents peuvent être plus ou moins ignorants. Et les propriétés des agents eux-mêmes, telles que leurs croyances et leurs désirs, sont également considérées comme intrinsèquement fixées ${ }^{17}$.

13. Ibid. p. 274.

14. Ibid., p. 281.

15. Ibid. p. 282.

16. Ibid. p. 283.

17. Ibid., p. 289. 
La conception objective et rationaliste des probabilités sous-entendrait que, bien que cela ne soit pas forcement et immédiatement connu par les agents, une estimation correcte et universalisable des décisions est disponible, et cela permet de satisfaire des utilités prédéterminées. Cela impliquerait, selon Bitbol, une contradiction, puisque la découverte d'utilités nouvelles et de nouvelles pratiques ne peut pas être prise en considération comme découlant de la rationalité qu'on se donne toute faite a priori. Ainsi, la seule manière cohérente d'envisager la connaissance consisterait à considérer que les conditions transcendantales de la connaissance, ce qui permet à un sujet de s'orienter d'une manière efficace dans le monde, sont le produit du processus d'énaction par lequel le pôle objectif et le pôle subjectif se co-instituent. De cette manière, les normes sont engendrées avec ce qui est normé dans un processus de cocréation continuel où l'apparaître et ses conditions se co-déterminent dans la vie imprévisible de la connaissance. La théorie de la décision conforme au corrélationisme radical n'implique pas des utilités données a priori avec une stratégie rationnelle toute faite, mais "les préférences et les objets se codéterminent dans le contexte de la situation de choix». La théorie des probabilités adaptée à cette théorie de la décision est celle utilisée par la physique quantique, notamment par le QBisme, c'est-à-dire une interprétation subjective plutôt qu'objective qui ne sous-entend aucun processus prédéterminé et indépendant de l'engagement pragmatique.

Bien avant son usage dans le cadre de la physique quantique, on disposait d'une épistémologie fondée sur l'interprétation subjective des probabilités développées à partir des travaux de De Finetti, Ramsey et Jeffrey, ce dernier proposant le susmentionné "probabilisme radical» étayé par un critère de cohérence fort différent de celui proposé par Bitbol. À la fois corrélationiste, antimétaphysique et pragmatiste, le probabilisme radical adopte une position anti-phénoménologique, bien qu'il soutienne: 1) l'idée qu'il n'y a pas une réalité en soi qui puisse se dire indépendamment d'un faire corrélé; 2) l'idée que le devenir de la connaissance est un processus imprévisible de co-constitution du réel. Il me semble que l'avantage du probabilisme radical est que l'on n'a aucunement besoin de postuler une instance fondatrice telle que la conscience et la notion ambiguë de totalité du monde, comme unités pré-subjectives, pré-rationnelles, et donc inconnaissables, dont parle Bitbol en s'exprimant par des énoncés que Wittgenstein définirait comme "philosophiques » et qui, à mon avis, laissent au matérialiste spéculatif la chance de l'emporter. En fait, une fois que cette totalité est admise comme idée, on ne voit pas pour quelle raison il faut la tenir pour nécessaire si on ne veut pas admettre une nécessité externe, comme un Dieu créateur l'ayant choisie parmi d'autres et dont la possibilité, au moins logique, ne peut pas être niée a priori. Comme Bitbol le dit, "cela pourrait ne pas être, et cela est; cela pourrait être autrement, et c'est ainsi; mais la pensée que cela pourrait être autrement n'est que l'écho affaibli et abstrait de la féérie de son "c'est ainsi". De cette ultime contingence, on ne peut rien inférer. " 
Cela veut dire que ce monde est contingent au moins parce que l'on ne saurait lui trouver une raison ou une cause externe: Meillassoux ne demande pas plus que cela, lorsqu'il soutient que la corrélation découvre, en son fondement, un manque de raison. Ainsi, d'autres totalités que celle dans laquelle nous sommes immergés deviennent, pour le matérialiste spéculatif, possibles au sens logique, c'est-à-dire ne contredisant aucun principe qui rend ce monde-ci la seule et unique réalité nécessaire, principe qui échappe aussi au corrélationiste radical. Il me semble donc que la seule manière pour défier le matérialisme spéculatif de Meillassoux est une position corrélationiste encore plus radicale que celle de Bitbol, une position qui puisse arriver à interdire tout énoncé métaphysique comme une hypothèse à laquelle il faut attribuer une probabilité $=0$, c'est-à-dire un degré zéro de croyance subjective, et donc, aucune signification pratique (c'est-à-dire qu'il ne serait pas rationnel de parier sur la possibilité de la réalisation, par exemple, du monde de justice divine dont parle Meillassoux). Or, parmi les énoncés à interdire, il y aurait aussi ceux qui portent sur la totalité vécue de la phénoménologie, cette réalité inconnaissable mais supposée comme fondement absolu du «s'apparaître du monde », c'est-à-dire de la raison de ce qui est sans autre raison que son "se co-déterminer dans un processus imprévisible». J'essayerai de montrer que, en renonçant aux résidus métaphysiques impliqués par la phénoménologie, le probabilisme radical est un meilleur candidat pour défier le matérialisme spéculatif, et qu'il pose également des défis concernant la cohérence effective du corrélationisme radical de Bitbol.

Selon l'approche de De Finetti, la probabilité est la mesure du degré de croyance subjectif et cela est complètement relatif, pour expliquer cela.

Supposons que l'on a observé de nombreuses fois que, après une éclipse, une guerre éclate. Pourquoi ne dis-je pas que l'éclipse est une cause de la guerre et pourquoi des superstitieux peuvent le croire ? et pourquoi les appeler superstitieux? En disant que l'éclipse est une cause de la guerre, je veux dire que, si demain je vois une éclipse, ce n'est pas pour autant que l'éclatement d'une guerre me semblera plus vraisemblable que si l'éclipse ne s'était pas vérifiée. En revanche, celui qui soutient que l'éclipse est une cause de guerre estime que, après une éclipse, une guerre est imminente. Je l'appelle superstitieux, car son état d'esprit diffère du mien et de celui de la civilisation à laquelle j'appartiens, car il désavoue la conception du monde qui est le fruit le plus intime de mon imagination et de l'imagination de mon siècle. Cependant, si je me défais pour un instant de ce qui, dans ma pensée, est une création à moi, si je veux distiller ce qui est objectif, c'est-à-dire purement logique ou purement empirique, dès lors je suis obligé de reconnaitre qu'il n'y a aucune raison de préférer mon état d'esprit à celui du superstitieux, sauf la raison que je le sens m'appartenir tandis que celui du superstitieux me dégoûte ${ }^{18}$.

De Finetti, ici, soutient que l'attribution de probabilité est un état mental éprouvé par le sujet et, en tant que tel, subjectif et non soumis à aucune 
contrainte a priori, ce qui rend acceptable toute stratégie de pari sur le futur à partir du présent de la croyance.

Il ne s'agit pas de rechercher la vérité mais seulement de devenir conscient de son opinion. Il ne s'agit pas d'interroger la nature, mais seulement d'examiner sa conscience. Au mieux, je peux interroger la nature afin qu'elle m'offre des données, tels que des éléments pour le jugement, néanmoins la réponse ne se trouve pas dans les faits; c'est dans mon esprit que les données, ne pouvant pas me conditionner, sont ce par quoi je me sens spontanément conditionné ${ }^{19}$.

La croyance éprouvée, on pourrait dire vécue, est celle des habitudes, c'està-dire des hypothèses d'action pratiques qui guident nos décisions, sans qu'il ne soit jamais possible d'opérer une telle réduction à dépasser, dans l'intimité de la conscience, la donnée en quoi consiste la probabilité qu'on attribue à quelque chose. Autrement dit, aucune vérité ne peut être atteinte par la réduction mais seulement le degré de croyance personnel, c'est-à-dire la probabilité qu'une hypothèse prédictive soit fiable, y comprise l'hypothèse de l'immersion dans le monde comme totalité réelle. Selon le probabilisme radical, rien ne peut être tenu pour certain, sauf la croyance que certaines opinions ou hypothèses fournissent des règles pratiques efficaces.

Qu'un évènement soit pratiquement certain, ou qu'il ne le soit pas, est une opinion et pas un fait; que moi je le juge pratiquement certain est un fait et pas une opinion. Que je doive agir en accord avec cette opinion n'est un corollaire que d'une façon apparente, car cette opinion subsiste parce que j'estime devoir régler mes actions en accord avec celle-ci ${ }^{20}$.

On peut croire que mes connaissances dérivent d'un processus d'interaction par lesquels les pôles subjectif et objectif se co-déterminent, mais la vérité est du côté du fait que je le crois parce que cela s'est avéré utile afin de faire des prévisions: le fait n'est pas la totalité vécue mais la croyance, ou la probabilité attribuée à cette hypothèse, laquelle peut être très proche de la certitude. En conséquence, le critère de cohérence requis par de Finetti est beaucoup plus économique que celui de Bitbol, puisqu'il suffit que les degrés de croyance permettent des paris tels qu'on puisse espérer de remporter le prix envisagé (lequel ne peut pas être établi universellement a priori). Ainsi, le critère de cohérence requis est que les croyances doivent respecter les lois de la probabilité.

Lorsqu'un individu a évalué les probabilités de certains événements, deux cas peuvent se présenter: ou bien il est possible de parier avec lui en s'assurant de gagner à coup sûr, ou bien cette possibilité n'existe pas. Dans le premier cas, on doit dire évidemment que l'évaluation de la probabilité donnée par cet individu contient une incohérence, une contradiction intrinsèque; dans l'autre cas, nous dirons que l'individu est cohérent. C'est précisément cette condition

19. Ibid., p. 19. Ma traduction

20. Ibid., p. 39. Ma traduction 


\section{Philosophiques / Automne 2020}

de cohérence qui constitue le seul principe d'où l'on puisse déduire tout le calcul des probabilités : ce calcul apparaît alors comme l'ensemble des règles auxquelles l'évaluation subjective des probabilités de divers événements par un même individu doit être assujettie si l'on ne veut pas qu'il y ait entre elles une contradiction fondamentale ${ }^{21}$.

De cette manière, de Finetti propose un critère de cohérence entre le dire, le degré de croyance subjectif en une hypothèse prédictive, et le faire, les décisions et les pratiques par lesquelles on s'oriente dans le monde, qui supporte les sciences contemporaines en proposant une réponse tout à fait pertinente au problème de l'induction, sans pour autant avoir besoin d'une épistémologie naturalisante - avec ses implications matérialistes et métaphysiques - afin de justifier l'accord intersubjectif et le processus d'évolution des savoirs. "En d'autres termes, il s'agit de considérer la coïncidence des opinions comme un fait psychologique; les raisons de ce fait pourront alors conserver leur nature subjective, qu'on ne peut laisser de côté sans soulever une foule de questions dont le sens n'est pas très clair ${ }^{22}$. " Nos hypothèses évoluent comme autant de stratégies de pari efficaces, et ces stratégies constituent, pourrait-on dire, les conditions transcendantales qui caractérisent une époque particulière, des croyances qui ne sont pas immunes de révision, puisque leur vérité se donne comme relative, située, plutôt qu'absolue. En adoptant les règles de Bayes pour réviser les croyances à la lumière des nouvelles informations collectées au cours de la pratique expérimentale, de Finetti complète la perspective épistémologique du probabilisme.

Une évaluation de probabilité n'a de sens que tant et jusqu'au moment où un individu ne connaît pas le résultat de l'événement envisagé; avant qu'il ne connaisse ce résultat (et qu'il soit ainsi amené à la valeur définitive o ou i), il pourra apprendre successivement plusieurs circonstances qui feront modifier son jugement, tantôt dans un sens et tantôt dans l'autre. Sans qu'il s'agisse là d'une correction ou d'un rejet. C'est de façon parfaitement identique que nous envisageons l'influence de l'observation sur la prévision dans le cas général des jugements fondés sur l'expérience ${ }^{23}$.

Le bayesianisme proposé par de Finetti, et développé par le probabilisme radical de Jeffrey, n'est pas la forme rationnelle critiquée par Bitbol, selon laquelle «il existe une distribution de probabilités (logique, a priori) qui définirait l'état d'esprit d'une intelligence parfaite, libre de toute expérience ${ }^{24}$ » au contraire,

21. Bruno de Finetti, "La prévision: ses lois logiques, ses sources subjectives ", Annales de l'Institut Henry Poincaré, tome 7, n. 1 (1937), p. 1-68, p. 7.

22. Ibid., p. 17.

23. Ibid., p. 55.

24. Richard Jeffrey, «Radical Probabilism (Prospectus for a User's Manual) », Philosophical Issues, vol. 2, Rationality in Epistemology (1992), p. 193-204, p. 196. Ma traduction. 
Le probabilisme radical ne cherche pas à analyser le jugement selon ses composantes purement rationnelles et purement empiriques, sans résidus. Il rejette le mythe empiriste de données sensibles de même que le mythe rationaliste de l'ignorance des priors; il rejette l'image du jugement comme une pièce de monnaie dont une face est empirique et l'autre rationnelle ${ }^{25}$.

Cette position implique que les données empiriques ne sont que des impressions subjectives qu'on décide de considérer comme se référant immédiatement à des objets externes pour des raisons pratiques, bien que ce soient les concepts des objets qui, comme des règles inductives, permettent de constituer les objets par l'attente des impressions. Comme le dit Bitbol: «Un objet est déclaré exister, selon la définition phénoménologique, si la structure d'attentes qui le constitue se trouve (provisoirement) confirmée par un événement sensible qui s'inscrit dans cette structure, c'est-à-dire par une impression qui la "remplit" 26 "soit, comme de Finetti l'explique:

Il est commode de pouvoir dire, d'une manière elliptique, «ce crayon existe, il est rouge, il est en bois » en sous-entendant le sujet «JE» qui exprime la phrase complète "Moi, je ressens cette sensation particulière de voir, toucher, ... que je caractérise par le mot "crayon", et, en outre, celles qui correspondent aux mots «rouge», "en bois». Tout cela est effectivement commode et aucun inconvénient logico-pratique dérive du fait que, en conséquence de la longue habitude à ces formes elliptiques, on oublie, et même on nie, leur caractère d'abréviation en les considérant comme des espèces de "vérités " indépendantes de nous, de nos sensations et de notre esprit ${ }^{27}$.

Mais pourquoi donc aurait-on besoin d'une épistémologie naturalisante et matérialiste, au sens métaphysique, puisqu'elle suppose une donnée certaine et fondatrice (se savoir être comme immergé dans une totalité s'apparaissant) —, lorsque pour expliquer l'évolution de la structure transcendantale on peut, d'une manière beaucoup plus simple, considérer le devenir des conventions linguistiques qui permettent de transmettre des règles d'inférence inductive sélectionnées pour leur efficacité ? Comme Jeffrey le note « les modes de jugement (probabiliste, etc.) et les standards de rationalité qui y sont associés sont des artefacts culturels, des ensembles de pratiques modifiés par la découverte ou l'invention de caractères conçus comme les supportant ${ }^{28}$ ». Pourquoi aurait-on besoin d'une explication naturaliste conduisant à ce que, du point de vue du probabilisme radical, cela soit une contradiction performative? Cette contradiction dépend du fait que, une fois admis l'interprétation subjective des probabilités endossée par les QBistes, on ne peut attribuer la certitude absolue à aucune de nos croyances, et donc, on n'est pas autorisé, comme Bitbol le suggère, à pos-

25. Ibidem. Ma traduction

26. Michel Bitbol, op. cit., p. 81.

27. Bruno de Finetti, «Probabilismo », cit., p. 63. Ma traduction.

28. Richard Jeffrey, «Radical Probabilism», cit., p. 203. Ma traduction 
tuler la vérité absolue du fait de se savoir vivant dans une totalité dont le concept est aussi flou qu'on ne saurait pas indiquer les impressions qu'il est censé subsumer. Comme les QBistes le remarquent, l'inférence inductive n'est pas plus qu'un jugement partagé et fondé sur l'habitude. L'habitude peut être modifiée par l'évolution, cependant elle reste une habitude ${ }^{29}$.

Les habitudes inductives n'ont pas besoin d'une autre justification que celle de leur efficacité pratique lorsqu'il s'agit de faire des prévisions impliquant des décisions, c'est-à-dire du critère de cohérence de De Finetti. Pour toute explication concernant la manière de faire évoluer les croyances et les théories scientifiques, on n'a besoin que des règles de Bayes. Cette position corrélationiste, laquelle refuse toute idée concernant une réalité indépendante de l'expérience et faisant de toute connaissance ce qui est fondé sur la croyance présente et ressentie par chaque individu historiquement, culturellement et socialement situé, permet de déclarer incohérent le pari de Meillassoux sur la possibilité de réalisation d'un monde autre que le nôtre du fait que, de son propre aveu, on ne saurait en estimer la probabilité. En reprenant le fameux pari de Pascal, on pourrait dire que se comporter comme si ce monde pouvait disparaître sans raisons en laissant la place au monde possible d'une justice divine, permettrait un gain infini bien que, du point de vue inductif et bayésien (celui adopté par Laplace dans sa critique de Pascal) on n'ait aucune raison d'y croire, puisque aucune information disponible ne supporte cette théorie. D'ailleurs, on pourrait se demander, et je trouve que c'est le point où Meillassoux et Bitbol s'accordent, s'il faut accepter que le principe qui supporte nos pratiques et notre croyance est la loi de satisfaction de l'utilité (utilité épistémique y comprise) dans le cadre du jeu évolutionnaire de l'économie globale dans lequel on est situé. Est-il nécessaire que nous jouions ce rôle d'agents stratégiques et croyions que la valeur des connaissances ne s'évalue que par le critère d'efficacité?

À ce propos, je voudrais attirer l'attention sur le fait que les probabilités subjectives font partie des outils développés afin de normer les comportements des agents économiques en offrant des hypothèses d'action auxquelles il faudrait croire afin de remporter son gain d'une manière dynamique, s'écartant donc du paradigme classique et objectiviste de la décision rationnelle portant sur des critères et des utilités données a priori. A-t-on le droit à l'incohérence (métaphysique), lorsqu'il s'agit de défier une cohérence inacceptable? Il me semble, en fait, que les matérialismes de Meillassoux et de Bitbol, qui portent sur les conditions réelles de genèse des êtres déterminés (d'une part la non-totalité de l'hyperchaos, de l'autre la totalité du monde de la vie), s'opposent tous les deux à l'idéalisme qui fait du réel la convergence de croyances dont la vérité se mesure en termes de rentabilité.

29. Christopher A. Fuchs, N. David Mermin, and Rüdiger Schack, «An introduction to QBism with an Application to the Locality of Quantum Mechanics, cit., p. 752. Ma traduction 